Attribution a Couperin le Grand. D'une pièce anonyme d'un recueil de Ballard Author(s): A.-M. D. Tessier

Source: Revue de Musicologie, T. 3, No. 2, [Papers Dedicated to François Couperin] (Jun., 1922), pp. 69-78

Published by: Société Française de Musicologie

Stable URL: http://www.jstor.org/stable/925635

Accessed: 11-06-2016 14:34 UTC

Your use of the JSTOR archive indicates your acceptance of the Terms \& Conditions of Use, available at

http://about.jstor.org/terms

JSTOR is a not-for-profit service that helps scholars, researchers, and students discover, use, and build upon a wide range of content in a trusted digital archive. We use information technology and tools to increase productivity and facilitate new forms of scholarship. For more information about JSTOR, please contact support@jstor.org.

Société Française de Musicologie is collaborating with JSTOR to digitize, preserve and extend access to Revue de Musicologie 


\section{ATTRIBUTION A COUPERIN LE GRAND \\ D'une pièce anonyme d'un recueil de Ballard}

Cette pièce, s'il est prouvé qu'il la lui faille attribuer, n'ajoutera que peu de choses à la gloire de François Couperin ; mais rien de ce maitre ne saurait manquer complètement d'intérêt.

Ballard publie en 1707 , un petit livre in-40 oblong, imprimé, de 28 pages, sous le titre: Pièceschoisies | pourleclavecin, | dedifferents auteurs. | Aucune adresse, aucune date, à ce titre, qui a ainsi toute l'apparence d'un faux-titre. Ces marques sont placées à la fin du volume. On lit à la dernière page : A Paris, | chez Christophe Ballard, seul Imprimeur du Roy pour la Musique, I ruë S. Jean de Beauvais, au Mont-Parnasse.| M.DCC.VII. | Avec Privilege de Sa Majesté. | (1)

Le livre est imprimé, avec les caractères ordinaires des Ballard, assez grossièrement, assez mal lisible. On mesure ici les raisons du triomphe, presque achevé à ce moment déjà, de la gravure, les raisons qui ont fait la décadence de l'antique maison des imprimeurs du Roi pour la musique. La gravure, malgré son prix élevé et les lenteurs de son emploi, se trouvait donner des résultats bien supérieurs à l'imprimerie pour la clarté de la présentation et de la lecture. L'imprimerie, surtout quand il s'agissait de figurer des accords ou plusieurs parties sur une même portée, dès qu'elle voulait travailler pour le clavier, non plus pour l'orchestre ou les voix, était maladroite.

Le volume contient seulement dix pièces, et les noms des auteurs ne sont pas donnés. Il est probable que nous avons là une de ces publications subreptices, établies sans la participation des auteurs, établies sur ces manuscrits des ouvrages en réputation qui couraient

(1) Exemplaire à la Bibl. Nat. sous la cote Vm7. 1861. 
le monde assez nombreux, et faisaient la célébrité d'un homme longtemps parfois avant qu'il ait rien donné de lui-même aux éditeurs. Quoiqu'il en soit, ces dix pièces ont été parfaitement choisies parmi celles qui avaient certainement le plus de succès à ce momentlà ; le nombre des manuscrits de l'époque qui les reproduisent prouve assez leur vogue ; et choisies d'autre part, la chose est très notable, dans la production la plus moderne, la production qu'on devrait dire avancée, celle qui commençait à mêler dans la musique française, plus retenue et plus décente d'ordinaire, les formes aux vives arêtes, aux chatoiements brillants, des sonates à l'italienne. Il est même à remarquer que la maison des Ballard, souvent accusée de n'avoir point suivi d'assez près les modes, d'avoir retardé les courants, se montre l§, bien au contraire, dans une posture d'avant-garde. Certes, rien ou presque rien, dans les autres livres de clavecin parus à la même époque (1), et ceux-là signés, ne présente encore ce style, celui de la seconde école du clavecin français, le style du Xviri ${ }^{\mathbf{e}}$ siècle, que Couperin en 1713 seulement, dans son $1^{\text {er }}$ livre gravé, fera paraitre tout armé de ses grâces frivoles et piquantes, tout frais, tout prêt pour une longue carrière. Le petit livre de Ballard est donc la première sortie en librairie de la nouvelle manière clavecinistique, et cela seul lui assure de l'importance historique, il manifeste avant tout autre le changement très net du goût public. Je ne dirai pas son progrès, car enfin tout n'est que changement.

Les noms des auteurs de la plupart des dix pièces publiées par Ballard, ne sont pas bien difficiles à découvrir. Quelques titres, d'abord, bien connus par ailleurs, attirent l'œil, et font diriger les recherches dans la bonne voie. Voici la liste de ces dix pièces, avec les attributions qu'il a été possible de faire :

Pièces en G. Ré. Sol.

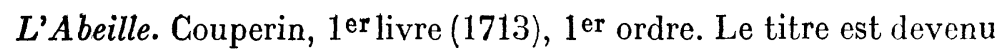
Les Abeilles.

Gavotle Italienne.

(1) Les deux petits livres de Marchand paru chez Ballard en 1702 ; ceux de Clérambault (1704), J. F. d'Andrieu (vers 1705), Gaspard le Roux(1705), Rameau (1706), Mile de la Guerre (1707), etc. 
Les Nonettes, avec ses deux parties, mineure et majeure : Les Blondes et les Brunes. Couperin $1^{\mathrm{er}}$ livre (1713), $1^{\mathrm{er}}$ ordre.

Sicilienne.

$$
\text { Pièces en D. La Re. }
$$

La Diane. Couperin. $1^{\mathrm{er}}$ livre (1713), $1^{\mathrm{er}}$ ordre.

La Florentine. Couperin. Ibidem.

\section{Pièces en $A . M i L a$.}

La Badine (en la majeur). Couperin. 1er livre, $1713,5^{\mathrm{e}}$ ordre.

La Badine (en la mineur). Attribuée à "M. Marchand d'Umaine ", par le ms. de transcriptions pour flûte de la Bibl. Nat. Vm 7 4866, p. 7.

\section{Pièces en $F$. Ut. Fa.}

La Polonaise. Marais, $2^{\mathrm{e}}$ livre de Viole (1701), pp. 55-56.

La Vénitienne. Attribuée à Marchand par le ms. Vm? 4866, p. 24, et par d'autres (1).

Comme on le voit, ces pièces ont presque toutes des titres caractéristiques, et non plus des titres de danses. Point d'Allemandes, de Courantes, ni de Sarabandes, mais des Badines, des Abeilles, des Vénitiennes, voire des Nonnettes. Bien que l'emploi de cette sorte de titres, en rapport avec les tendances générales et constantes de la musique française, ne soit point une invention, ni même une spécialité de Couperin, il s'est beaucoup généralisé de son temps et sous son influence: Ainsi la présence exclusıve de ces titres dans le livre de Ballard marque bien que les pièces qu'il contient sont de la nouvelle façon.

Les cinq qui sont de Couperin sans conteste, et que leur publication en 1707 chez Ballard n'a pas empêché d'être replacées par leur auteur lui-même dans son 1 er livre, sont présentées dans un texte à peu près entièrement conforme au texte définitif de 1713. Iln'y a, à

(1) Cf. Ertner. Article Louis Marchand; et dans les Archives des $M^{\mathrm{e}^{\mathrm{s}}}$ de l'Orgue. Tome V, la seconde notice de M. Pirro sur Marchand. L'Air de la Venitienne avec le nom de Marchand et des paroles parodiées, est même dans un ouvrage gravé, les Nouvelles Poésies Spirituelles et Morales sur les plus beaux airs... $3^{\mathrm{e}}$ Recueil (1732), p. 34 . 
ce qu'il m'a semblé, entre les deux versions, que des variantes sans grande importance, dont les principales portent sur les agréments qui, l'on pouvait s'y attendre, sont beaucoup plus diversifiés et mieux précisés dans l'édition donnée par Couperin. Cette publication de Ballard a du moins l'intérêt de nous prouver que ces pièces étaient composées bien avant 1713. On s'en doutait; on se doutait qu'une bonne part des pièces du livre de 1713 avaient eu leur naissance plus tôt; la date de composition de certaines d'entre elles doit remonter jusqu'aux dernières années du $\mathrm{xvII}^{\mathrm{e}}$ siècle. Couperin, en effet, était célèbre bien avant l'édition de son 1er livre, et célèbre spécialement comme maitre et compositeur de clavecin. En 1713, il a déjà 45 ans, il y a 20 ans qu'il a l'honneur "d'estre au Roy"; c'est un homme arrivé, dont on recherche les ouvrages en manuscrit depuis déjà longtemps. Il nous le dira, parlant à ses acheteurs : "Il m'a êté impossible de satisfaire plûtôt les désirs du public en luy donnant mes piéces gravées : j'espere qu'il ne me soupçonnera pas d'avoir affecté ce retardement pour piquer d'avantage sa curiosité... On sçait assés qu'vn auteur n'a que trop d'interest de donner vne edition corecte de ses ouvrages, lors qu'ils ont eu le bon-heur de plaire : s'il est flaté par les aplaudissemens des connoisseurs, il est mortifié par l'ignorance, et les fautes des copistes, c'est le sort des manuscrits recherchés... " (1). Au reste, publiant tard, et seulement après le succès décidé de ses œuvres, Couperin ne fait qu'imiter ses prédécesseurs, Chambonnières, Le Bègue, d'Anglebert et les autres, qui, la plupart, ont attendu le plus qu'ils ont pu avant de se décider à faire graver.

Les deux pièces de Marchand ont ce grand intérêt de nous montrer un Marchand fort différent de celui qui, en 1702, publiait deux livrets de pièces de clavecin, entièrement, et de bon cœur, rattachées à l'ancien style. Marchand jeune, rapidement célèbre à Paris comme organiste, Marchand à la quête de la gloire qui lui sourit, et soucieux de lui faire rendre au plus tôt profit, n'a point encore l'idée de rien changer aux formes traditionnelles. Ses̀ deux suites de 1702 sont parmi les publications de clavecin de cette époque celles peut-être qui sont le plus proche des vieux livres de Chambonnières et de

(1) Préface des Pièces de Clavecin composées par M. Couperin. $1^{\text {ex }}$ Livre. 1713, in-folio. 
Le Bègue, ce qui ne les empêche pas d'ailleurs d'avoir des mérites notables et personnels. Mais ce Marchand, sans cesse célèbre, le grand Marchand dont la renommée alla croissant, ou du moins toujours égale, jusqu'à la fin de.sa vie, comme il dédaignait sans doute après 1702 une gloire trop vite maîtrisée, ou, de toutes façons, que ce soit à cause de difficultés qui tiennent à sa vie privée, ou bien par une bizarrerie d'esprit que ses contemporains lui reprochèrent, Marchand n'a plus rien voulu publier. Nous ne saurions donc point, n'étaient ces deux pièces (1), que, de très bonne heure, il a suivi Couperin, son rival, dans la nouvelle voie, où vraiment il l'a serré de près. Car ces deux morceaux ont bien autant de brio, de grâce tendre et d'aisance mélodique équilibrée, qu'un bon nombre de pièces de l'inventeur du nouveau style, que toutes celles que les contemporains peut-être estimèrent le plus, c'est-à-dire les moins complexes d'inspiration et de rédaction.

Pour la Vénitienne, l'attribution à Marchand certifiée par plusieurs documents ne fait pas de doute. Pour la Badine en la mineur, la chose n'est pas aussi claire. Le manuscrit de transcription pour flûte que je citais, dit : " de M. Marchand d'Umaine. " Je n'ai pas vu que Louis Marchand se soit jamais fait appeler ainsi, mais jusqu'à preuve du contraire, il y a lieu de croire que c'est de lui qu'il s'agit. Cette Badine, comme les autres pièces du recueil de Ballard, se retrouve dans plusieurs manuscrits, et généralement accolée, comme soudée à la Badine en la majeur du 5 e Ordre de Couperin (2). Il semble qu'elle a été considérée comme en étant la suite, la seconde partie, comme elle paraît être dans le volume de Ballard, et qu'on la jouait d'ordinaire après la première Badine, et précédant une reprise de cette même première Badine. Coupe très fréquemment usitée à cette époque, et caractéristique du style de Couperin, que la division d'un morceau en deux parties majeure et mineure, ou mineure et

(1) N'étaient aussi quelques morceaux de chant parus dans les recueils de Ballard ou restés manuscrits, par exemple ce bel air italien, si brillant et si vide, Io provo nel cuore, qui fut fameux vers cette époque, et dans lequel, au dire de Fresneuse, les chanteurs de par delà reconnaissaient avec confiance le génie de leur cher pays. (Comparaison, Ed. de Hollande, tome III, p. 94).

(2) Le Père Pingré, par exemple, dans ses copies de pièces de Clavecin (Bibl. Sainte-Genev. mss. 2377 à 2381), l'intitule : "Badine servant de $2^{\mathrm{e}}$ partie à celle de M. Couperin. (Table de ces copies au ms. 2.381.) Dans un autre manuscrit, celui en ma possession dont je parlerai à propos de la Sicilienne, on lit : "La Badine de $M$. Couprin et la Suitte. . 
majeure, sur des thèmes différents mais ayant une analogie, avec reprise de la première partie pour finir. Peut-être cette particularité des deux Badines, l'une de Couperin, et l'autre qui n'est pas de lui, doit-elle rassurer notre conscience historique quand elle prononce le jugement d'une autre affaire de paternité musicale, celle qui se rapporte à la pièce les Bergeries du $2^{\mathrm{e}}$ Livre et $6^{\mathrm{e}}$ Ordre de Couperin ? On sait que Marchand, au dire du moins de certains contemporains, aurait prétendu que ces Bergeries étaient de lui-même, et que Couperin se les était sans vergogne appropriées (1). Cependant ledit Couperin n'a pas fait paraitre dans son 1 er livre, à côté de sa Badine en la majeur, cette autre Badine mineure que beaucoup considéraient comme étant de la sienne le complément naturel. Et si cette $2^{\text {de }}$ Badine était bien, comme il semble, une enfant abandonnée dans le monde sans l'aveu ni l'appui d'un père certain, et puisque, d'autre part, on avait l'habitude de l'apparenter à la première, qui aurait empêché Couperin, s'il n'eût pas été l'honnêteté même, de faire bien facilement pour elle comme on prétend qu'il ait fait pour les Bergeries, de l'adopter sans bruit?

La dernière pièce dont l'auteur est identifié est la Polonoise, de Marais. A son propos, Ballard a commis une erreur assez grosse. Cette pièce, soi-disant en F. Ut. Fa, est en réalité, dans cette version, pour clavecin comme dans celle que Marais avait déjà donnée pour la Basse de Viole, en ré mineur (D. La. Ré). Les adaptations pour un instrument différent de pièces originairement écrites pour un autre, étaient des choses constamment et librement faites. La plupart des auteurs de pièces instrumentales prévoyaient ces adaptations, les provoquaient même. La raison en est fort simple, c'est que si leur musique pouvait être jouée par les amateurs de différents instruments, il y avait plus de chance de voir les livres qui la contenaient prendre un grand débit. Il ne faut pas chercher plus loin l'explication d'habitudes qui ne sont pas d'abord sans nous choquer. Marais, par exemple, lui-même, dans l'Avertissement de son second Livre de viole de 1701, celui qui présente la Polonoise: On a eu attention, dit-il, en composant ces pièces, "de les rendre propres pour toutes sortes d'instrumens comme l'Orgue, Clavecin, Theorbe, Luth, Violon, Flutte Allemande. "Il en a fait l'épreuve sur ces deux der-

(1) Cf. Ch. Bouvet. Les Couperin, p. 54. 
niers. Et encore dans son 3e livre de 1711 : il est à propos d'avertir le public que la plupart des pièces de ce $3^{\mathrm{e}}$ livre «se peuvent joüer sur d'autres instrumens, comme l'orgue, le clavessin, le violon, le dessus de viole, le theorbe, la guitarre, la flutte traversiere, la flutte à bec, et le hautbois. Il ne s'agira que d'en sçavoir faire le choix pour chacun de ces instrumens ". Et aussi de savoir faire ces adaptations. Il est intéressant de voir comment a été faite celle de la Polonoise pour le clavecin, haussant le chant presque partout à l'octave au-dessus pour donner plus de place aux accords de la basse chiffrée, trop resserrés et gênés si l'on avait gardé, avec la tessiture peu élevée de la basse de viole, ces grands sauts de dixièmes ou de douzièmes dans le grave qu'elle avait la possibilité et l'habitude de faire. On ne peut savoir si c'est Marais lui-même qui a écrit cette adaptation. La chose serait assez vraisemblable. En tout cas, le travail a été bien fait, et cette Polonoise est certainement dans le recueil de Ballard une des meilleures pièces, et riche en solide harmonie (1). Marais d'ailleurs, " un des illustres de nos jours ", comme dira Couperin (2),et l'un de ses meilleurs rivaux dans la musique instrumentale de son temps, Marais dont le labeur fervent a produit, je crois bien, près de 800 pièces, en trio ou pour la viole, et dont les livres s'échelonnent sur près de quarante années, de 1686 à 1720 passés, Marais est un musicien de grande classe. La présence d'une pièce de lui, composée en 1701 au plus tard, dans le recueil de 1707 dont le style est en général au goût nouveau, ne doit même pas nous surprendre: elle n'y fait pas disparate, et Marais en effet, dans sa production de longue durée parcourant la période même où le goût a changé, n'a jamais retardé trop sur la mode ; il est plutôt de ceux qui, avec Couperin, ont servi et guidé le mouvement. Parti au temps du plus pur lullisme, élève de Lully, dont il se vante, il a, sans en être le moins du monde effarouché, traversé l'époque des sonates, et gouverné toujours aisément sans toucher les écueils. Son goût naturel probablement le portait dans la voie nouvelle, sa facile veine mélodique, autant que sa science harmonique bien assise.

(1) On la retrouve comme les autres dans des manuscrits du temps : Bibl. Nat. Vm7 4872, p. 38, avec le nom de Marais. - Vm7 4866, p. 48, sans ce nom. - Vm7 6296 (ms. de viole.) p. 44, sans ce nom. - Ste-Genev., 2381, p. 340, sans ce nom. - Ms. en ma possession p. 56 , avec ce nom.

(2) Préface du 2e Livre de Clavecin, sans date (1717). 
Il reste deux pièces auxquelles on a pu remarquer que je n'ai pas donné de noms d'auteurs. Pour la Gavotte italienne, en sol, je n'ai rien rencontré jusqu'ici. On la retrouve dans divers manuscrits, mais toujours anonyme (1). Le hasard fera-t-il qu'un jour ou l'autre l'auteur en soit trouvé ? Pièce d'ailleurs dont l'intérêt n'est pas exceptionnel, pièce brillante mais un peu vide. Peut-être, comme le titre pourrait l'indiquer, faudrait-il chercher du côté des maîtres italiens, peut-être est-ce là simplement une adaptation de clavecin de la gavotte d'une de ces sonates qui faisaient fureur à Paris à l'époque où Ballard a publié ce morceau ? Sinon, c'en est du moins un pastiche évident. En voici le début :

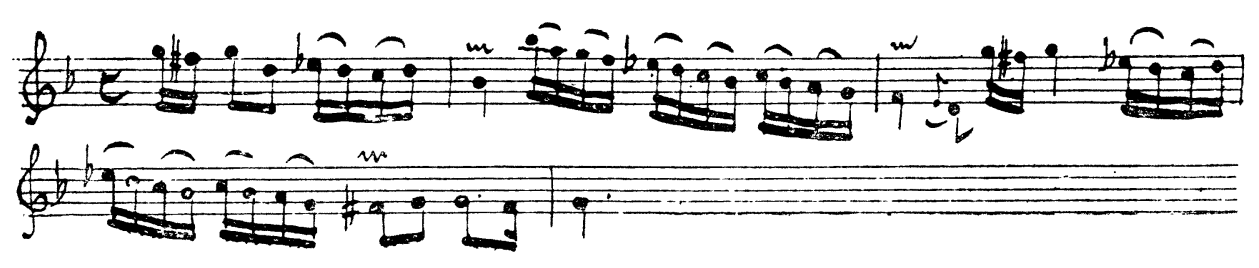

La Sicilienne est celle dont, sur la foi d'un manuscrit, je propose l'attribution à Couperin. C'est une pièce fréquemment ausssi rencontrée manuscrite (2), une de celles du recueil de Ballard, conséquemment, qui paraissent avoir eu le plus de vogue. Mais je n'ai vu jusqu'ici qu'un seul recueil, depuis peu en ma possession, qui marque un nom d'auteur. Il l'intitule : «Sicilienne de M. Couprin» (3). C'est un manuscrit rédigé probablement par un amateur de chant et de violon, qui n'a transcrit que le dessus d'un bon nombre de pièces vocales et instrumentales, des premières années du $\mathrm{XVIII}^{\mathrm{e}}$ siècle, avec quelques-unes antérieures, des brunettes et des airs célèbres de Lully. La date de la composition de ce recueil doit être aux environs de 1710 (4). Les attributions qu'il fait paraissent

(1) Sainte-Geneviève, 2381, p. 337 - ms. en ma possession, p. 64, etc.

(2) $\mathrm{Vm}^{7} 4866$, p. 32- Sainte-Genev., 2381, p. 338. - Dans un exemplaire des Basses-continues du $1^{\mathrm{er}}$ Livre de Viole de Marais (B. N. Vm ${ }^{7} 6267$ ) on l'a copiée sur les pages de garde de la fin, en dessus seul.

(3) P. 88.

(4) Les pièces de Couperin qu'il contient sont toutes du Ier Livre (1713) et probablement recueillies avant l'édition, car l'une d'elles, l'Abeillle, y porte ce titre qu'elle a dans le volume de Ballard, et non pas les Abeilles comme dans le 
exactes : on y voit des pièces dites de Couperin, Marais, Forcroy, Du Val, De Caix, et qui sont bien en effet de ces auteurs. Beaucoup d'autres sont sans nom, mais les auteurs de certaines peuvent facilement être déterminés.

Une attribution faite par un seul manuscrit ne peut pas être considérée comme tout à fait sûre. Il arrivait aux rédacteurs de ces recueils de se tromper ; assez souvent des pièces passaient, dans un petit groupe de gens qui se croyaient informés, pour être d'un auteur qui n'était pas le vrai. Dans le cas présent, il reste surprenant que Couperin, qui a replacé dans son 1 er Livre, les cinq autres pièces de lui parues dans le volume de Ballard, n'ait point jugé à propos d'y mettre aussi cette Sicilienne, ni de la mettre dans aucun de ses livres publiés ensuite, de clavecin, de pièces en concert, ou de "sonades". Car, sauf erreur de ma part, elle ne se retrouve en nul endroit de ses livres gravés. Néanmoins je serais porté à ne pas trop contester cette attribution. L'on peut remarquer qu'en somme, c'est une pièce dans la manière de Couperin, du moins sa manière courante et sans recherche, celle-là qui a le mieux fait son succès. C'est une mélodie alerte, coulant d'abondance, un thème qui a presque un air de déjà entendu, sans qu'on puisse préciser le souvenir qu'il éveille, et puis une harmonisation sans aucune complexité assurément, et ce rythme aux temps bien scandés occupés par des groupes de trois croches, qui est celui $(12 / 8,6 / 8,3 / 8,12 / 16$, etc. $)$, de toutes les Siciliennes, Vénitiennes, et autres pièces de ce temps qui affichent par ces titres leurs origines incontestables. Couperin a tant d'autres pièces de la même façon, qui ne font point sa meilleure gloire, mais après tout n'y nuisent pas (l'auteur des Charmes, des Baricades Mistérieuses, et des Ombres Errantes, n'a d'ailleurs point à craindre de déchoir), il en a tant qu'on ne voit point pourquoi celle-ci ne serait point de lui. A défaut de la transcription complète, nous allons reproduire le dessin qui forme le thème principal de cette Sicilienne;

livre gravé. İl y à cependant dans ce ms. (p. 90) une pièce qui n'a paru que bien plus tard dans un livre publié en 1733. C'est la Badine de d'Agincour, en mi majeur, une $3^{\mathrm{e}}$ Badine. Elle fait partie du $4^{\mathrm{e}}$ ordre de d'Agincour, mais elle est ici dans un autre ton (sol majeur) que celui qu'elle a dans le livre de 1733, et sous un tout autre titre : le Papillon, rondeau. Tout cela doit nous indiquer que la date de composition de cette pièce est très antérieure à 1733, d'Agincour a d'ailleurs commencé sa carrière de compositeur et d'organiste dès les dernières années du $\mathrm{xvII}^{\mathrm{e}}$ siècle. 
après quoi, et à titre de comparaison, nous en ferons autant pour la Florentine, elle aussi parue d'abord dans le petit livre de Ballard, mais que Couperin n'a pas négligé de remettre dans son édition de 1713.
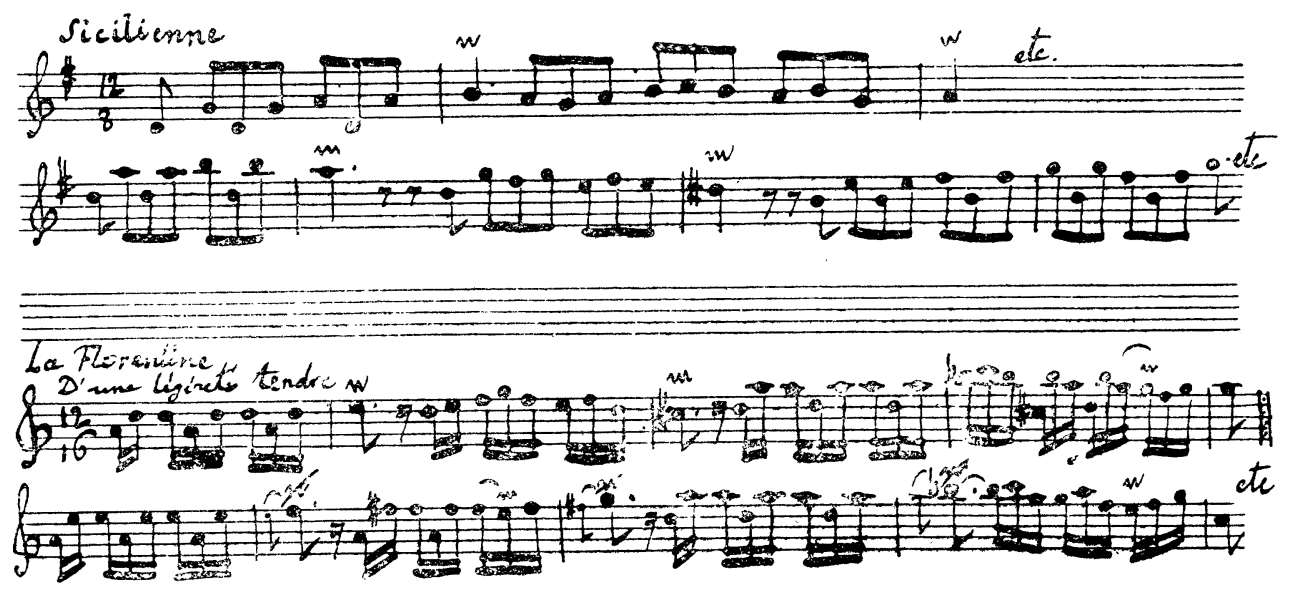

Les thèmes et la manière dont ces thèmes sont traités ont bien de l'analogie, et vraiment cette Florentine et cette Sicilienne, malgré l'éloignement prétendu de leurs lieux de naissance, se ressemblent, comme deux filles nées d'un même père.

A. Tessier.

Erratum. - P. 76 ci-dessus, dans la citation musicale, ajoutez un dièse devant le $f a, 1^{\text {re }}$ note de la $3^{\mathrm{e}}$ mesure. 